\title{
Study of Incidence of Microalbuminuria Among First Diagnosed Diabetic Patients and its Correlation with Body Mass Index and Coexisting Hypertension in A Tertiary Care Hospital
}

\author{
${ }^{1}$ Esakki Muthuvel, Vimal Chander $\mathbf{R}^{1 *}$ and Sowmiyabalu ${ }^{2}$ \\ 'Department of Pathology, Saveetha Medical College and Hospital, Thandalam, Chennai, India \\ ${ }^{2}$ Tagore Medical College and Hospital, Rathinamangalam, Candour Post, Chennai. India
}

\begin{abstract}
Background: Diabetes is raising recently more in the developing countries due to sedentary life style and with increase in duration, complications arise affecting renal, retinal, cardiovascular and nervous systems. Diabetic nephropathy poses a great risk of renal failure and is the cause for performing dialysis. Microalbuminuria is the earliest marker for the renal and cardiovascular involvement. This study aims to study the incidence of microalbuminuria in first diagnosed diabetic patients and to correlate it with the body mass index (BMI), hypertension and waist hip circumference.
\end{abstract}

Methods: This study was conducted on 138 first diagnosed diabetic patients in a tertiary care hospital. Microalbuminuria was analyzed using dipstick method and correlation was made with parameters such as blood pressure, body mass index, and waist-hip circumference.

Result: The incidence of microalbuminuria in first diagnosed diabetic patients was $17 \%$. Incidence of microalbuminuria is increased in hypertensive diabetic patients $(37 \%)$ compared with non-hypertensive diabetic patients $(10 \%)$, which was statistically significant $(\mathrm{p}=0.001$, $\mathrm{r}=0.292$ ). Incidence of microalbuminuria among first diagnosed diabetic patients was $17 \%$. The correlation of microalbuminuria with hypertension, BMI and waist-hip ratio suggest that microalbuminuria incidence is significantly associated with hypertension than BMI and waist-hip ratio.

Conclusion: Microalbuminuria is the earliest indicator for detecting nephropathy. The incidence of microalbuminuria of $17 \%$ during first diagnosis of diabetes indicates the inadequacy in the health care system regarding the screening programs in the rural population. Thus, improvements must be made in the health care system to reduce the incidence rate by effective screening program.

Keywords: Microalbuminuria, Diabetes Mellitus, Body Mass Index, Hypertension, Type 2 Diabetes.

\section{Introduction}

Diabetes is a rising crisis in India. India leads the world with the largest number of diabetic subjects and this is expected to further rise in the coming years. Hence studies on diabetes related complications are essential to assess the burden of diabetes. ${ }^{[1]}$ The prevalence of diabetes is increasing sharply in the developing countries as people adopt more sedentary life styles. Type 1 and 2 diabetes mellitus have caused a great havoc by increasing the morbidity and mortality by mainly affecting the cardiovascular, renal, retinal system, nervous system and others. ${ }^{[2]}$

Focusing on its effect on the renal system, the main etiology being due to angiopathy of renal capillaries, which leads to chronic renal disease, which is the prime indicator for dialysis. In initial stage, the kidney may leak slightly more albumin than normal amount in urine, which can be sensitively detected by albumin specific dipstick test for albumin, which is known as microalbuminuria. Microalbuminuria is the earliest indicator of diabetic kidney disease and generalized vascular endothelial dysfunction. [3] The microalbuminuric patients had a significantly higher prevalence of ischemic heart disease compared with normoalbuminuric patients. Retinopathy was also common among the microalbuminuric group. In patients with known diabetes, microalbuminuria is related not only to subsequent proteinuria, but also even more strongly to early death, mainly from cardiovascular disease.

Diabetic nephropathy is the leading cause of end stage renal disease worldwide. Diabetic nephropathy is defined by persistent albuminuria (albumin excretion rate $>300$ $\mathrm{mg} /$ day), declining glomerular filtration rate and rising blood pressure. ${ }^{[4]}$ Microalbuminuria is considered to be an early stage of diabetic nephropathy. Nephropathy is probable cause of the elevated blood pressure found in diabetic patients. It has been suggested that in both Insulindependent diabetes mellitus (IDDM) and Non-insulindependent diabetes mellitus (NIDDM), hypertension increases the chances of development of diabetic 
nephropathy. ${ }^{[5]}$ In NIDDM, microalbuminuria prevalence ranges from 15 to $20 \%$ and nephropathy often supervenes after a shorter duration of diabetes than in IDDM. ${ }^{[4]}$ The causal risk factors for microalbuminuria are raised blood pressure and poor glycemic control and achievement of optimal glycemic level reduces the risk of albuminuria and microalbuminuria. This calls for early detection of microalbuminuria and good control of diabetes to reduce the burden of diabetic complication in the future.

The present study aims to study the incidence of microalbuminuria in first diagnosed diabetic patients and to correlate it with the body mass index (BMI), hypertension and waist-hip circumference.

\section{Materials and Methods}

This cross-sectional study was conducted at Tagore Medical college and hospital in Chennai over a period of 2 months from July to August 2013, wherein suspected patients were diagnosed to have diabetes by random blood sugar test and subsequently confirmed by fasting \& postprandial blood sugar values. All first diagnosed diabetic patients are included in this study, while patients with significant proteinuria, urinary tract infection, or heart failure were excluded from this study. In all 138 patients who were included in this study, a complete clinical work up was done including height, weight, and body mass index, waist hip ratio. The body mass index (BMI) was calculated and the patients are classified as normal (BMI <25), overweight (BMI $>25 \&<30)$ and obese (BMI $>30)$. The blood pressure was recorded in the right upper arm in the sitting posture, after a five-minute rest. Mean of two blood pressure measurements was used for analysis. Patients were categorized as hypertensive if they were on antihypertensive treatment or in the presence of systolic blood pressure $>140 \mathrm{mmHg}$ and/or diastolic blood pressure $>90 \mathrm{mmHg}$. Waist circumference was measured midway between the lower rib and iliac crest while that of the hip at the level of trochanters and the ratio was calculated. The patients are classified as two groups based on the waist hip ratio as group 1 (male $<1$; female $<0.9$ ) and group 2 (male $>1$; female $>0.9$ ). Then spot urine collection is collected from the patient and checked for microalbuminuria. Urine microalbumin concentration was tested using albumin specific dipstick method. In case of dehydrated patients and for those after heavy exercise, urine sample is collected during their next visit to the hospital. Statistical analysis were done using SPSS software version 16 . The results were given by median and standard deviation. Correlation was made between microalbuminuria and parameters such as blood pressure, body mass index, and waist hip circumference.

\section{Result}

A total of 138 first diagnosed type 2 diabetic patients were included for this study, which included 46 males and 92 females. Overall 24 patients (17\%) had microalbuminuria (Figure 1). Among males, microalbuminuria was positive for 6 among $46(13 \%)$ and for females it is 18 among $92(20 \%)$.

Hypertensives: Total number of diabetic patients with hypertension was 38 out of 138. Among the 38 hypertensive diabetic patients 14 had Microalbuminuria and among 100 non-hypertensive diabetic patients 10 had microalbuminuria. The incidence is $37 \%$ and $10 \%$ amongst the Hypertensive diabetic and non-Hypertensive Diabetic patients respectively (Figure 2). The incidence of microalbuminuria is increased in hypertensive patients compared to non-hypertensive patients. The Incidence of Microalbuminuria among Hypertensive Diabetic patients in those with only raised systolic blood pressure is 2 among $13(15 \%)$ or only raised diastolic blood pressure is 2 among $5(40 \%)$ or both is 10 among $20(50 \%)$ (Figure 3). The statistical association between microalbuminuria and hypertension is $\mathrm{p}=0.001, \mathrm{r}=0.292$. These results suggest that diastolic blood pressure is correlated to microalbuminuria more than systolic blood pressure.

Body Mass Index (BMI): Incidence among the patients with normal BMI, overweight, obese are found to be 10 in 63,8 in 55 and 6 in 20 i.e. $16 \%, 15 \%, 30 \%$ respectively (Figures 4 and 5). There are no significant changes regarding incidence of microalbuminuria among the overweight patients and those with normal BMI, but it is significantly increased among the obese patients. Statistically association between Body Mass Index and microalbuminuria is $\mathrm{p}=0.6$ and $\mathrm{r}=0.04$, hence $\mathrm{BMI}$ is not directly associated with microalbuminuria.

Waist hip ratio: Based on the waist hip ratio patients are classified as Group 1 (male $<1$; female $<0.9$ ) and Group 2 (male $>1$; female $>0.9$ ) and incidence among the group 1 and group 2 is 13 among 93 (14\%) and 11 among $45(41 \%)$ respectively (Figure 6). Though microalbuminuria appears to be incident more among the person with high waist hip ratio, but statistically the association between waist hip ratio and microalbuminuria is $\mathrm{p}=0.138, \mathrm{r}=0.127$ hence this relation was insignificant.

Anyway, difference in incidence of microalbuminuria regarding body mass index and waist hip circumference suggests that its incidence differs among different parameters of obesity index. Microalbuminuria was present in $41 \%$ of hypertensive diabetic patients with BMI $>25$, it was present in $37 \%$ of hypertensive diabetic patients with BMI $<25$. 


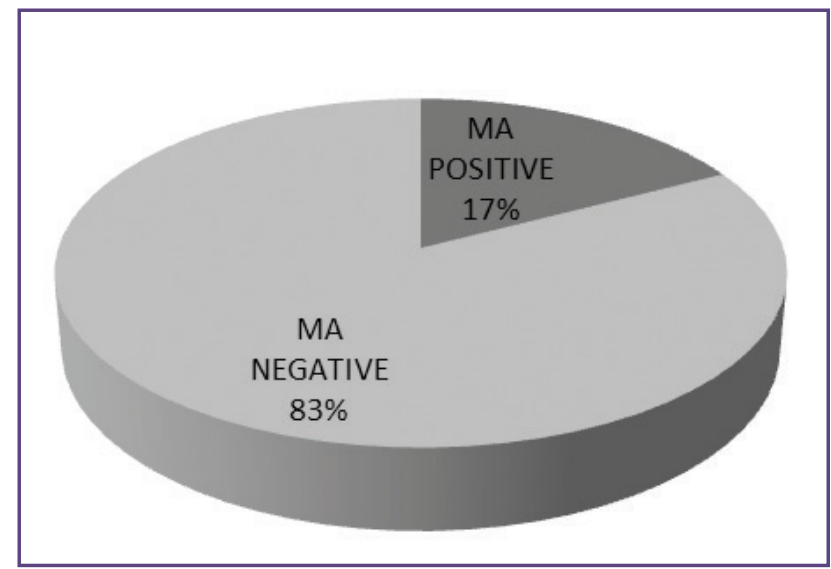

Fig. 1: Incidence of microalbuminuria among first diagnosed diabetic patients.

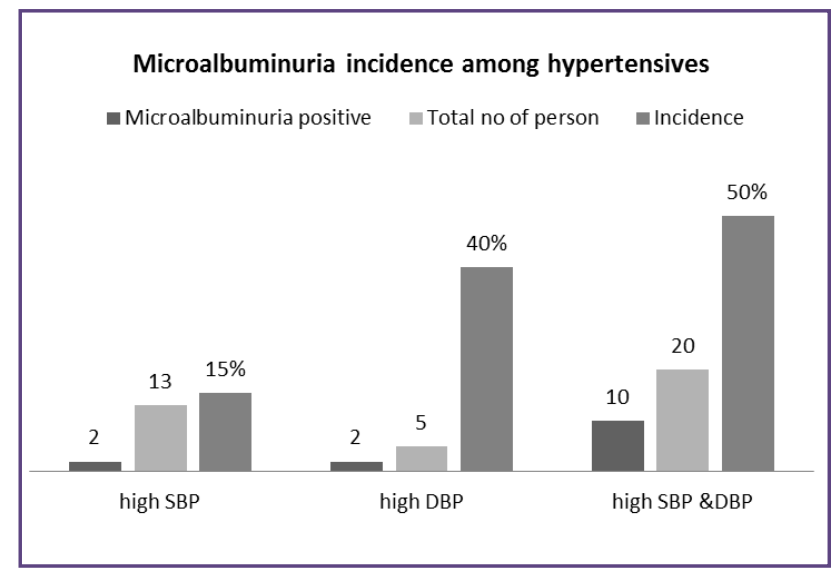

Fig. 3: Incidence of microalbuminuria among persons with only high systolic blood pressure (SBP), only high diastolic blood pressure (DBP) and those with both.

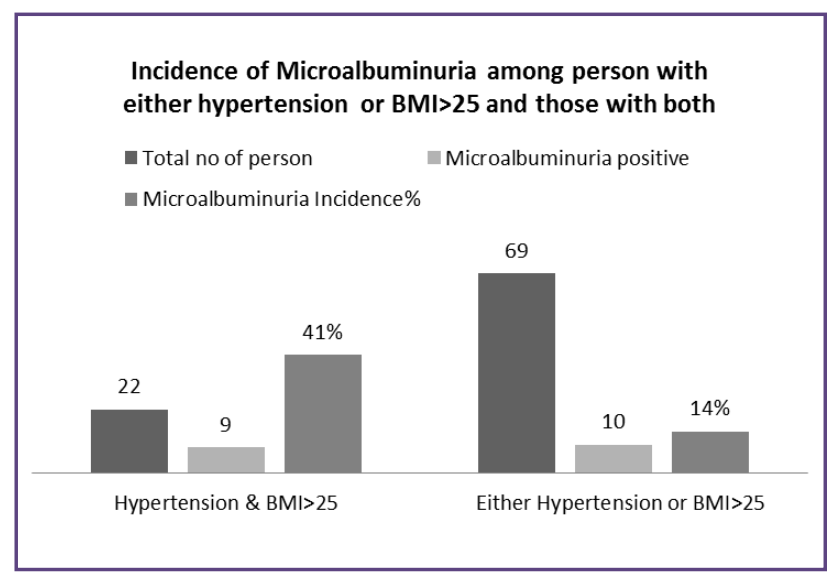

Fig. 5: Microalbuminuria incidence among person with either body mass index (BMI) $>25$ or hypertension and those with both.

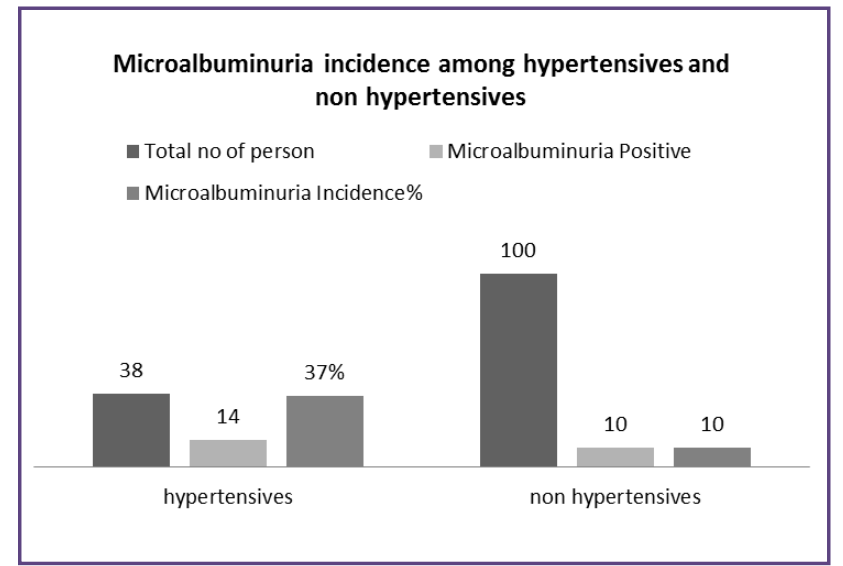

Fig. 2: Incidence of microalbuminuria among hypertensives and non-hypertensives.

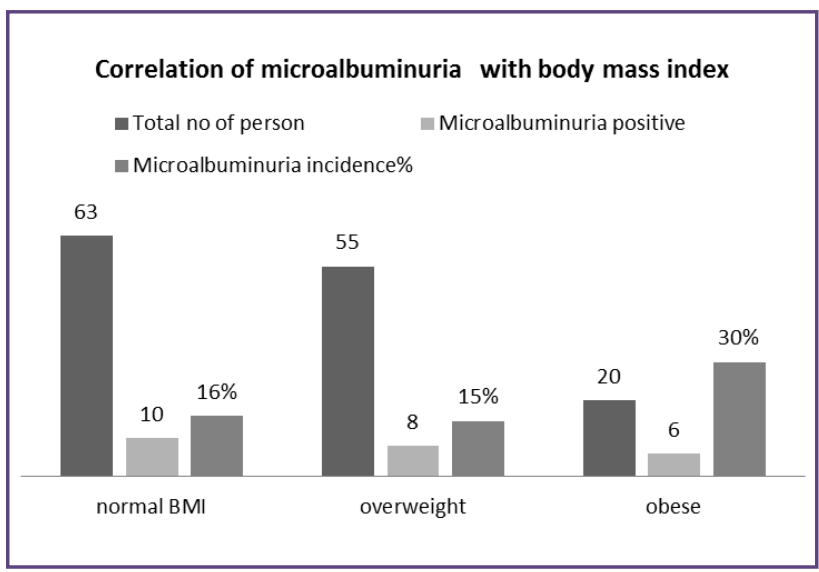

Fig. 4: Incidence of microalbuminuria among person with normal $\mathrm{BMI}(<25)$, overweight $(\mathrm{BMI}>25 \&<30)$ and obese (BMI $>30$ ).

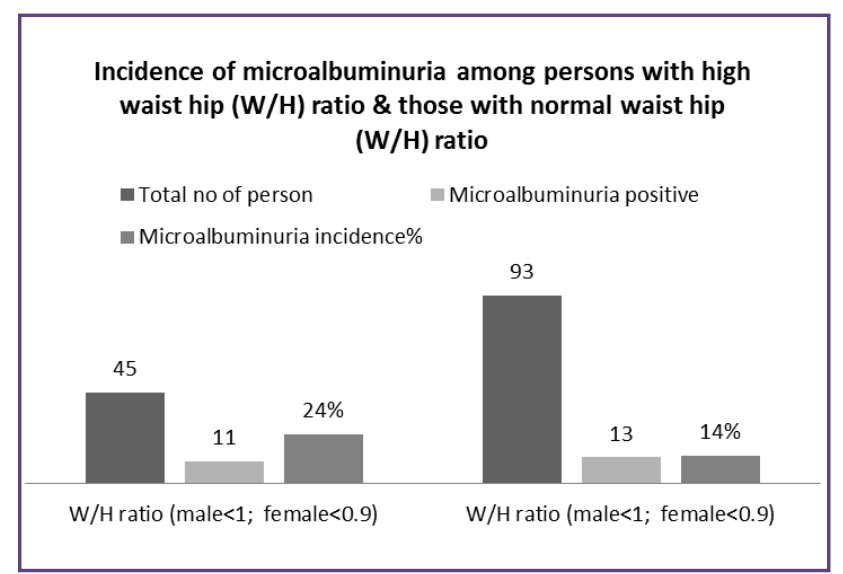

Fig. 6: Incidence of Microalbuminuria among persons with high waist hip $(\mathrm{W} / \mathrm{H})$ ratio and those with normal waist hip $(\mathrm{W} / \mathrm{H})$ ratio. 


\section{Discussion}

Incidence of microalbuminuria in the initially diagnosed diabetic patients in this study was $17 \%$ by dipstick method, whereas the incidence among newly diagnosed diabetic patients using immunoturbidometric test was $27 \%$ in a study conducted in South India. ${ }^{[1]}$ The difference in the incidence may be due to large sample size and the sensitivity and specificity of immunoturbidometric assay compared to the albumin specific dipstick method. The prevalence of microalbuminuria increased with the increase in duration of diabetes. Altogether $27.5 \%$ of the newly diagnosed diabetic subjects had microalbuminuria by immunoturbidometric assay kits. At the time of diagnosis of diabetes, 24 among 138 patients had microalbuminuria which is a significant and is an opportunity to find the involvement of kidney at the most early stage and taking measures to prevent its progression. Among the other parameters measured, it is probably evident that incidence of microalbuminuria is increased among hypertensive diabetic patients i.e. 14 among 38 having $37 \%$ of chances than in non-hypertensive patients i.e. 10 among 100 having a chance of $10 \%$. The result on statistical correlation $(\mathrm{p}=0.001, \mathrm{r}=0.292)$ between hypertension and microalbuminuria also found to be significant. This also correlates with many studies about the incidence of microalbuminuria among the hypertensive diabetic patients. ${ }^{[6,7,8]}$

In this study, the incidence of microalbuminuria among patients with $\mathrm{BMI}<25$; BMI $>25 \&<30$ and those with BMI $>30$ is $16 \%, 15 \%$ and $30 \%$ respectively. Statistical association of Body mass index and microalbuminuria is $\mathrm{p}=0.6$ and $\mathrm{r}=0.04$, hence Body mass index is not directly associated with microalbuminuria. Though obesity is a general risk factor for cardiovascular problems and many others, there is no statistical significance of incidence of microalbuminuria among the overweight and obese population. In a study conducted among 25 patients with body mass index $>25$, Blood pressure and urinary albumin excretion rate in hypertensive patients with obesity significantly decreased with weight reduction. ${ }^{[9]}$ Hence there may indirect relevance of microalbuminuria and body mass index. And further studies in this topic may widen our knowledge and fill the gap in the understanding.

The incidence of microalbuminuria among those with high waist hip ratio was $24 \%$ were as those with moderate waist hip ratio was $14 \%$. Though there was striking incidence of microalbuminuria in patients with abdominal obesity compared to general obesity, there is no statistical correlation $(\mathrm{p}=0.138, \mathrm{r}=0.127)$ between microalbuminuria and waist-hip ratio in our study. In a multinational, observational study, 20828 hypertensive out-patients from 26 countries had participated and the suggested that increasing Waist circumference confers an incremental risk for microalbuminuria at any level of BMI, underlining the prognostic importance of abdominal fat accumulation beyond general obesity. ${ }^{[10]}$ This deviation result may be due to small group of people involved in our study or due to short term follow up or only hypertension is strongly correlated to microalbuminuria when compared to diabetes.

Testing strategies involving dipstick and laboratory Albumin creatinine ratio (ACR) measurements or dipstick tests had similar accuracy. The costs of using dipstick tests were overall lower than laboratory ACR-based testing. ${ }^{[11]}$ Anyway of the urine screening tests, the dipstick test is inexpensive, easy and rapid to perform, des not delay testing the microalbuminuria, since there is no wait for the screening test. Diabetic patients with micro- or macroalbuminuria should be carefully controlled in order to prevent or to decrease deterioration of renal function due to diabetic nephropathy. Hence steps should take for the early detection of microalbuminuria and to prevent further involvement of the kidney giving more preference to the hypertensive diabetic patients since there are at high risk.

\section{Conclusion}

Incidence of microalbuminuria is significantly associated with hypertension than body mass index and waist hip ratio. Microalbuminuria is the earliest detector for the kidney involvement as well as cardiovascular involvement and screening for it in the earliest stage helps to reduce the occurrence of nephropathy. The incidence of microalbuminuria of $17 \%$ during first diagnosis of diabetes indicates the inadequacy in the health care system regarding the screening programs in the rural population. Thus improvements must be made in the health care system to reduce the incidence rate by effective screening program.

\section{Reference}

1. Varghese A, Deepa R, Rema M, Mohan V. Prevalence of microalbuminuria in type 2 diabetes mellitus at a diabetes centre in southern India. Postgrad Med J 2001;77:399-402.

2. Mogensen CE. Preventing end-stage renal disease. Diabet Med 1998;15 Suppl 4:S51-6.

3. Kong NC, Chia YC, Khalid BA, et al. Microalbuminuria prevalence study in hypertensive type 2 diabetic patients in Malaysia. Med J Malaysia 2006 Oct;61(4):457-65.

4. Lehmann R, Spinas GA. Diabetic nephropathy: significance of microalbuminuria and proteinuria in Type I and Type II diabetes mellitus. Praxis. 1995;84(44):1265-71.

5. Valensi P, Ferrière F, Attali JR, Erault C, Modigliani E, Delrieux C, Sebaoun J. Microalbuminuria in diabetics with moderate hypertension. Arch Mal Ceur Vaiss. 1986;79(6):785-9. 
6. Zarini GG, Exebio JC, Gundupalli D, Nath S, Huffman FG. Hypertension, poor glycemic control, and microalbuminuria in Cuban Americans with type 2 diabetes. Int J Nephrol Renovasc Dis 2011;4:35-40.

7. Vasović O, Zamaklar M, Lalić K, Milosević D, Zikić L, Popović L. The prevalence of hypertension and microalbuminuria in diabetes mellitus type 1 and type 2 . Srp Arh Celok Lek. 2005;133(5-6):229-32.

8. Descamps O, Buysschert M, Ketelslegers JM, Hermans M, Lambert E. Microalbuminuria in a population of 653 patients with type 1 and 2 diabetes. Diabete Metab 1991;17(5):469-75.

9. Ohashi H, Oda H, Ohno M, Watanabe S. Weight reduction improves high blood pressure and microalbuminuria in hypertensive patients with obesity. Nihon Jinzo Gakkai shi 2001; 43(4):333-339.

10. Thoenes M, Reil JC, Khan BV, et al. Abdominal obesity is associated with microalbuminuria and an elevated cardiovascular risk profile in patients with hypertension. Vasc Health Risk Manag. 2009;5(4):577-85.

11. Nagrebetsky A, Jin J, Stevens R, et al. Diagnostic accuracy of urine dipstick testing in screening for microalbuminuria in type 2 diabetes: a cohort study in primary care. Fam Pract. 2013 Apr;30(2):142-52.

12. Mur Martí T, Franch Nadal J, Morató Griera J, Llobera Serentill A, Vilarrubias Calaf M, Ros Espin C. Nephropathy and microalbuminuria in type II diabetes. Aten Primaria. 1995;16(9):516-24.
13. Weir MR. Albuminuria predicting outcome in diabetes: incidence of microalbuminuria in Asia-Pacific Rim. Kidney Int Suppl. 2004 Nov;(92):S38-9.

14. Gupta DK, Verma LK, Khosla PK, Dash SC. The prevalence of microalbuminuria in diabetes: a study from north India. Diabetes Res Clin Pract 1991 May;12(2):125-8.

15. Lutale JJ, Thordarson $\mathrm{H}$, Abbas $\mathrm{ZG}$, vetvik $\mathrm{K}$. Microalbuminuria among Type 1 and Type 2 diabetic patients of African origin in Dar Es Salaam, Tanzania. Vetvi BMC Nephrol 2007;8:2.

16. Hoffmann IS, Jimenez E, Cubeddu LX. Urinary albumin excretion in lean, overweight and obese glucose tolerant individuals: its relationship with dyslipidaemia, hyperinsulinaemia and blood pressure. J Hum Hypertens 2001 Jun;15(6):407-12.

17. Rossi MC, Nicolucci A, Pellegrini F, et al. Identifying patients with type 2 diabetes athigh risk of microalbuminuria: results of the DEMAND (Developing Education on Microalbuminuria for Awareness of reNal and cardiovascular risk in Diabetes) Study. Nephrol Dial Transplant. 2008;23(4):1278-84.

18. Viswanathan M, Snehalatha C, Bhattacharyya PK, Mohan V, Ramachandran A. Microalbuminuria in NIDDM patients in south India. Indian J Med Res 1991;94:125-9.

19. Lum G. How effective are screening tests for microalbuminuria in random urine specimens? Ann Clin Lab Sci 2000;30(4):406-11.

*Corresponding author:

Dr. Vimal Chander R, 216, Mahatma Gandhi 2nd Street, Thiruverkadu Cooperative nagar, Chennai - 600077. India

Phone: +91 9790645729

Email: rvimalchander@gmail.com

Date of Submission : 04.01.2017

Date of Acceptance : 30.01.2017

Financial or other Competing Interests: None.

Date of Publication : 15.04.2017 